\title{
Genipin-Modified Silk-Fibroin Nanometric Nets
}

\author{
Simone S. Silva, ${ }^{*}$ Devid Maniglio, Antonella Motta, João F. Mano, Rui L. Reis, \\ Claudio Migliaresi
}

Nanometric silk-fibroin nets were fabricated by electrospinning from regenerated Bombyx mori silk-fibroin (SF)/formic acid solutions with the addition of genipin (GE), 2, 15 and $24 \mathrm{~h}$ after the solution preparation. After spinning, the pure fibroin nanofibers were water soluble and needed a further stabilization process, whereas the reaction of fibroin with genipin resulted in water-insoluble fibroin nets due to conformational changes induced in the fibroin by the genipin. SFGE nanofibers presented diameters ranging from 140 to $590 \mathrm{~nm}$ and were generally thinner than pure SF nanofibers. The secondary structure of SF into SFGE nanofibers showed the presence of a $\beta$-sheet conformation together with $\beta$-turn intermediates (turns and bends). The approach described in this paper provides an alternative method of designing SF nanofibers that are already water insoluble, without any stability post-treatment needed.

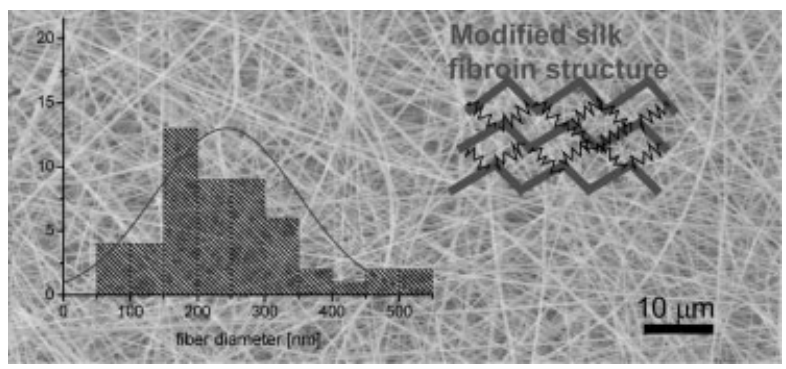

\section{Introduction}

Natural hierarchical organization at the nanolevel scale has been inspiring materials scientists in the design of novel nanofiber biomaterials for improving functionality and mechanical performance. The production of nanofibers from biopolymer solutions has gained a great deal of attention for biomedical uses, since the high-porosity electrospun mats that are formed by ultrafine fibers with a

S. S. Silva, J. F. Mano, R. L. Reis

3B's Research Group - Biomaterials, Biodegradables and Biomimetics, Department of Polymer Engineering, Campus de Gualtar, 4710-057 Braga, Portugal

Fax: +351 253604 4498; E-mail: simonesilva@dep.uminho.pt S. S. Silva, J. F. Mano, R. L. Reis

IBB - Institute for Biotechnology and Bioengineering, PT

Government Associated Laboratory, Braga, Portugal

D. Maniglio, A. Motta, C. Migliaresi

Department of Materials Engineering and Industrial Technologies and INSTM Research Unit, Via Mesiano 77, University of Trento, I - 38050 Trento, Italy high specific surface can mimic the structure of native extracellular matrices (ECMs). Furthermore, interactions between cells and an ECM are crucial to cellular differentiation and in modulation of cell function. ${ }^{[1,2]}$ The electrospinning technique has been recognized as an efficient, rapid and inexpensive method of manufacturing nanoscale fibrous structures for a number of biomedical applications. ${ }^{[3,4]}$ In the electrospinning process, a high voltage is applied to create electrically-charged jets of a polymer solution. As the conical jet of polymer fluid propagates through the air, the solvent evaporates and nanofibers are collected on the target as a nonwoven matrix. ${ }^{[2,3]}$ This technique has been utilized to generate nonwoven mats of nanometer-sized fibers from biopolymers. ${ }^{[5-11]}$ Among them, silk fibroin (SF), the natural biomacromolecule spun by the Bombyx mori silkworm has been used in the preparation of matrices (for example, tissue-engineered scaffolds, films, nanofibers) for a range of biomedical applications, due to its unique properties such as good biocompatibility, good oxygen and watervapor permeability and minimal inflammatory reac- 
tion. ${ }^{[9,10,12-15]}$ Nonwoven matrices of electrospun nanofibers have been obtained from a regenerated SF solution using different spinning solvents. ${ }^{[9-11,16,17]}$ However, electrospun SF nanofibers as well as others types of SF matrix, such as films, are water soluble and should be treated with an aqueous alcohol solution ${ }^{[13,15,17]}$ or with water vapor ${ }^{[11]}$ to enhance their crystallinity and stability in the presence of water. The main objective of this work was to obtain modified SF nanofibers stable in an aqueous environment, in a single processing step. This paper reports the first attempt to use genipin (GE) to modify regenerated SF solutions for the electrospinning process. Actually, genipin is considered a natural cross-linking agent with a lower cytotoxicity as compared with alternative cross-linkers such as glutaraldehyde. ${ }^{[18]}$ It has been reported that genipin binds with biological tissues $^{[19,20]}$ and biopolymers, ${ }^{[18,21]}$ leading to matrices with good mechanical properties, reduced swelling extent and good biocompatibility. Our aim was to investigate the effect of the addition of genipin to SF solutions on the processability, morphology and stability of electrospun fibroin nets. In fact, an increase of both the water-resistant ability and the mechanical performance of the resulting silk nanofibrous membranes could facilitate their practical applications. Properties of GE added fibroin solutions, structural changes and the morphological features of electrospun SFGE nanofibers were examined using rheological measurements, attenuated total reflection Fourier transform infrared (ATR-FTIR) spectroscopy and scanning electronic microscopy (SEM).

\section{Experimental Part}

\section{Materials}

Silk from cocoons of Bombyx mori was kindly provided from Cooperativa Socio Lario, Como, Italy. Genipin (GE) was a product of Wako Chemicals, USA. All of the other chemicals were reagent grade and used as received.

\section{Preparation of Silk Fibroin}

Silk from cocoons of Bombyx mori was first degummed in order to remove sericins. Degumming was achieved by boiling the silk filaments for $1 \mathrm{~h}$ in water containing $1.1 \mathrm{~g} \cdot \mathrm{L}^{-1}$ of $\mathrm{Na}_{2} \mathrm{CO}_{3}$ (anhydrous) and then for $30 \mathrm{~min}$ in water with $0.4 \mathrm{~g} \cdot \mathrm{L}^{-1}$ of $\mathrm{Na}_{2} \mathrm{CO}_{3}$. Finally, the resulting fibroin filaments were extensively rinsed in hot distilled water and dried in the air at room temperature. To prepare solutions to be used in the preparation of the materials, fibroin was dissolved in $9.3 \mathrm{M} \mathrm{LiBr}$ overnight at $65^{\circ} \mathrm{C}$. The solution was then dialyzed in a Slide-A-Lyzer Cassette (Pierce), (molecular-weight cut-off (MWCO) $=3500 \mathrm{Da}$ ) against distilled water for $3 \mathrm{~d}$ at room temperature. The water was changed every $2 \mathrm{~h}$. After that, the silk fibroin water solution was frozen in liquid nitrogen and lyophilized to obtain pure fibroin powder. The amino-acid composition of silk fibroin was obtained by HPLC analysis.

\section{Preparation of Spinning Silk-Fibroin Solution}

Regenerated silk fibroin (SF) dissolved in neat formic acid at concentration of 12 wt.- $\%$ was used. Genipin powder (12\%, related to the total weight of fibroin in solution, that is, $6.29 \times 10^{-3} \mathrm{M}$ ) was added to the silk-fibroin solution under stirring conditions for $5 \mathrm{~min}$ at room temperature until complete dissolution. The system was maintained under stirring for a predetermined time $(2,15$ and $24 \mathrm{~h})$ at $37^{\circ} \mathrm{C}$ and then the conductivities of the solutions were measured using a 525 CRISON conductimeter instrument. Subsequently, the SF solutions were submitted to the electrospinning process. The identification of the modified SF solutions was SFGEX, where GE and $X$ indicate genipin and the reaction time, respectively. As control materials, SF and SFGE films were also prepared by casting the solutions on Petri dishes. After casting, the films were dried at room temperature for at least two days before any further use.

\section{Production of Nanonetworks by the Electrospinning Process}

In the experimental electrospinning, the SF solution was loaded into a $20 \mathrm{~mL}$ syringe and a needle was attached. The positive electrode of a high-voltage power supply (Gamma High Voltage Research Inc.) was directly connected to the needle to charge the spinning drop of protein solution, and the negative electrode was clipped in the down part of the system. The syringe was placed on an automatic syringe pump to maintain a constant volume-flow rate. The electric potential, solution flow rate, and the distance between the needle tip and the aluminium foil were adjusted till a stable jet was obtained. The operating parameters selected to produce the nanofibers were the following: voltage $=21 \mathrm{kV}$, flow rate $=0.005 \mathrm{~mL} \cdot \mathrm{min}^{-1}$, distance between the needle tip and the target (aluminium foil set at ground voltage) $=10 \mathrm{~cm}$ and room temperature. The entire apparatus was mounted under a hood and nitrogen gas was insufflated continuously around the system during the electrospinning process to control the humidity, which was regulated to a range of $20-30 \%$. After preparation, all of the samples were dried in vacuum for 1 week prior the analysis.

\section{Characterization}

Rheological measurements were carried out on Advanced Rheometric Expansion System (ARES, Shear Strain Controlled). The geometry was a stainless-steel plate/plate (diameter $2 \mathrm{~mm}$ and gap $0.4 \mathrm{~mm}$ ). The plate was equipped with a solvent trap to reduce evaporation during measurement Dynamic rheological tests (time sweep) at frequency of $5 \mathrm{rad} \cdot \mathrm{s}^{-1}$ and strain of $5 \%$ were used to characterize the behavior of the solution before and after addition of genipin at $37^{\circ} \mathrm{C}$.

The electrospun nanofiber morphology was observed under a scanning electron microscope (SEM) (Cambridge Stereoscan 100) operated at an acceleration voltage of $10 \mathrm{kV}$. In the SEM photos, the fiber diameters of the nanofibrous membranes were analyzed 
using the ImageJ image-visualization software developed by the National Institute of Health (http://rsb.info.nih.gov/).

The mats obtained by electrospinning were cut into squares of $2 \times 2 \mathrm{~cm}$ and immersed in distilled water at $37^{\circ} \mathrm{C}$ for $8 \mathrm{~d}$ to test their dissolution/stability.

The infrared spectra of SF and SFGE nanofibers and films were obtained using Perkin Elmer and Bio-rad FTS6000 spectrometers, respectively, in the spectral region of $4000-650 \mathrm{~cm}^{-1}$. Curve fitting of the bands was performed with the ORIGIN ${ }^{\circledR}$ (OriginLab Corporation, MA, USA) software: Gaussian bands shapes were employed in this procedure. Curve-fitting was limited to the $1580-1720 \mathrm{~cm}^{-1}$ spectrum range, since the protein structure (contributions of the second and third vibration) can be identified in this range. ${ }^{[22,23]}$

\section{Results and Discussion}

\section{Silk-Fibroin-Solution Characterization}

Amino-acid composition of the fibroin used in this work was assessed by HPLC analysis. The silk-fibroin molecule was formed mainly of glycine (46.7\%), alanine (31.4\%), and serine $(8.5 \%)$. Prior to the preparation of nonwoven
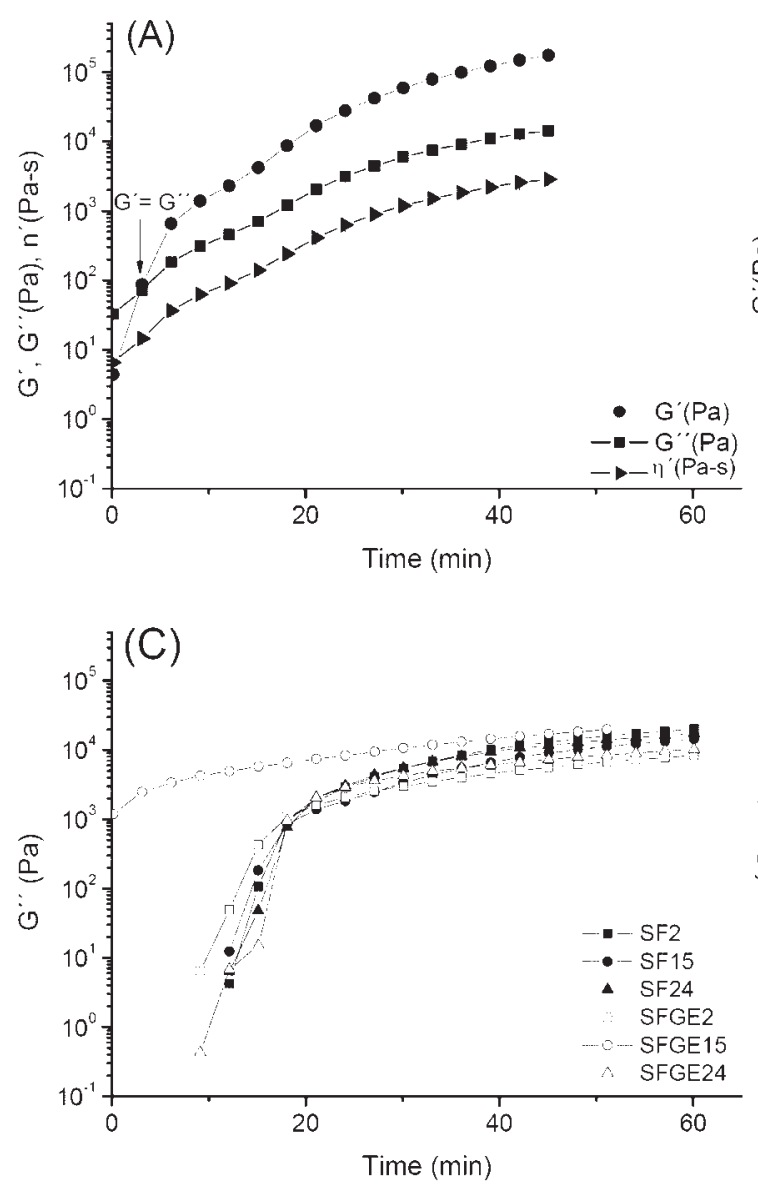

silk-fibroin mats by electrospinning, the behavior of the silk fibroin solutions after addition of genipin was examined by rheology measurements (Figure 1A). Both the storage shear modulus, $G^{\prime}(t)$, and loss shear modulus, $G^{\prime \prime}(t)$, were found to increase with time, until $G^{\prime}$ became greater than $G^{\prime \prime}$ due to formation of a protein network structure. The gel point, defined when $G^{\prime}=G^{\prime \prime}$, was observed after $3.5 \mathrm{~min}$. However, the stiffness of the gel kept increasing for longer times. Based on such results, SF solutions were electrospun 2, 15 and $24 \mathrm{~h}$ after preparation. Furthermore, dynamic time-sweep studies were performed on these SF and SFGE solutions. The rheological data of all of the SF solutions showed that $G^{\prime}(t), G^{\prime \prime}(t)$ and $\eta^{\prime}(t)$ values increase with time (Figure $\left.1(B-D)\right)$. This effect could be due to a slight gelation of the fibroin solution induced by the applied shear stress, a phenomenon well known for fibroin solutions. Moreover, values of $G^{\prime}(t), G^{\prime \prime}(t)$ and $\eta^{\prime}(t)$ were higher for the SFGE15 solution as compared with SFGE24, thus indicating the occurrence of some degradation at higher times.

The electrical conductivities of the SFGE solutions were higher than that of the regenerated SF solution (Table 1).
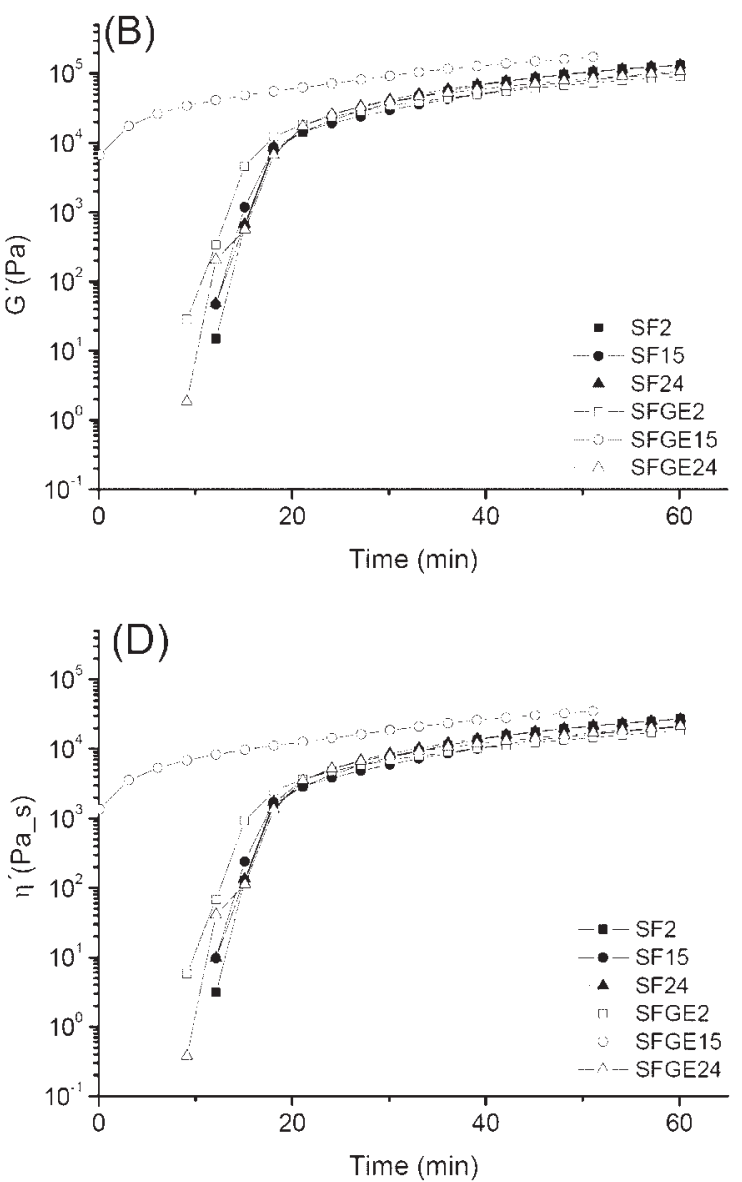

Figure 1. (A) Storage shear modulus $G^{\prime}(t)$, loss shear modulus $G^{\prime \prime}(t)$ and viscosity of SFGE solutions; (B) time dependence of storage shear modulus, $G^{\prime}(t)$, (C) loss shear modulus, $G^{\prime \prime}(t)$, and $(D)$ viscosity, $\eta^{\prime}(t)$, of SF and SFGE solutions after different reaction times. Measurements were made at $\omega=5 \mathrm{rad} \cdot \mathrm{s}^{-1}$ at $37^{\circ} \mathrm{C}$. The closed $(\boldsymbol{\square}, \mathbf{O})$ and open $(\square, \bigcirc, \triangle)$ symbols correspond to SF and SFGE solutions, respectively. 
Table 1. Characteristics of the SF and SFGE solutions and average fiber diameter of the SF nanofibers obtained before and after water-stability tests.

\begin{tabular}{|c|c|c|c|c|}
\hline \multirow[t]{2}{*}{ System } & \multirow[t]{2}{*}{$\begin{array}{l}\text { Color of the } \\
\text { solution }\end{array}$} & \multirow{2}{*}{$\frac{\text { Conductivity }^{\text {a) }}}{\mu \mathrm{S} \cdot \mathrm{cm}^{-1}}$} & \multirow{2}{*}{$\begin{array}{l}\begin{array}{c}\text { Fiber diameter before } \\
\text { water-stability tests }\end{array} \\
\mu \mathrm{m}\end{array}$} & \multirow{2}{*}{$\begin{array}{l}\begin{array}{l}\text { Fiber diameter after } \\
\text { water-stability tests }\end{array} \\
\mu \mathrm{m}\end{array}$} \\
\hline & & & & \\
\hline SF & Pale rose & 874 & $480 \pm 140$ & $900 \pm 280$ \\
\hline SFGE2 & Pink & 892 & $590 \pm 170$ & $1020 \pm 370$ \\
\hline SFGE15 & Dark pink & 925 & $240 \pm 110$ & $380 \pm 120$ \\
\hline SFGE24 & Dark red & 1160 & $140 \pm 70$ & - \\
\hline
\end{tabular}

The higher conductivity, resulting in more charges accumulated in the electrospinning jet, associated with the viscoelastic behavior of the modified SF solutions, promoted the formation of a more-stable jet and the production of nanofibers with smaller average diameters (Figure 2). Nevertheless, SF nanofibers were obtained only when the humidity of the system was controlled in the range of $20-30 \%$, using nitrogen gas. Higher humidity, in fact, could slow down solvent evaporation, thus hindering the formation of nanofibers. ${ }^{[3]}$

\section{Morphological Characterization}

SEM micrographs (Figure 2) show that as-spun SF and SFGE nanofibers were randomly distributed in the mats with an average fiber diameter that varied from 140 to $590 \mathrm{~nm}$ (Table 1). Also, the morphology of the SF nanofibers changed from a uniform fiber structure (Figure 2(A1-C1)) to fibers linked together with beads (Figure 2D1). The high conductivity of the SFGE solutions (Table 1) might increase the stretching of the polymer solution during electrospinning, thus resulting in small-diameter fibers. ${ }^{[3]}$ It is evident that the properties of the SFGE solutions (viscosity, conductivity) had a clear influence on both the morphology and the average diameter of the resulting fibers. Additionally, it is known that genipin reacts with amino acids or proteins to form dark-blue pigments. ${ }^{[19]}$ After mixing, the color of SFGE solutions changed gradually from a transparent pale rose to dark red (Table 1), thus indicating the progress of the reaction between the SF and GE. This fact also suggests that the reaction mechanism involving genipin and silk fibroin with formic acid as a solvent may be different to those reported in the literature. ${ }^{[18,24]}$

The water-stability of the SF nanofibers prepared with different reaction times (2, 15 and $24 \mathrm{~h}$ ) was evaluated through immersion of the nonwoven nets in distilled water at $37^{\circ} \mathrm{C}$ for $8 \mathrm{~d}$. As expected, the SF nanofibers without any stabilization post-treatment (Figure 3A1), lost their integrity, as reported in others studies. ${ }^{[11]}$ Fibers at junctions were fused together forming agglomerates on the surface (Figure 3A1), probably due the gradual dissolution of the material. Compared with the SF nanofibers, SFGE2 preserved its original fibrous form (Figure 3B1). Of all the fibers, SFGE15 showed the highest stability in water, with a small increase of the fiber diameter (Figure 3C1, Table 1). The interaction mechanism of genipin with silk fibroin is currently unknown; some authors ${ }^{[20]}$ have reported that genipin reacts preferentially with the amino acids, lysine and arginine, of proteins. In our studies, the fibroin chain contains those amino acids in a very low percentage (lysine $-0.6 \%$ and arginine $-0.6 \%$ ), mainly in the hydrophilic blocks. For this reason, the reaction kinetics should be reasonably slow, but efficient enough to render the system stable in water.

\section{FTIR-Spectroscopy Results}

Fibroin protein exists in three conformations, namely random coil, silk I ( $\alpha$-form), and silk II ( $\beta$-sheet conformation). SF in formic acid exhibits the random-coil conformation, similar to the aqueous SF solution. ${ }^{[25]}$ It is hypothesized that the SF molecules could structurally rearrange due to changes in the hydrogen bonding caused by the presence of the genipin, as well as by the processing effect. In order to evaluate the effect of genipin on the final conformation of fibroin, FTIR spectra of SF and SFGE nanofibers and films were acquired (see Figure 4). Even though new peaks did not appear after the reaction, structural changes can be revealed by analyzing the peaks due to amide I ( $\mathrm{C}=\mathrm{O}$ stretching) and II ( $\mathrm{N}-\mathrm{H}$ deformation) in the spectra (Figure $4 \mathrm{a}$ and $4 \mathrm{~b}$ ). Comparing the spectra acquired for both samples, the amide I (1 640-1 $620 \mathrm{~cm}^{-1}$ ) and amide II (1 529-1 $517 \mathrm{~cm}^{-1}$ ) bands, which are associated with the antiparallel $\beta$-structural conformation, ${ }^{[22,23]}$ shifted to lower wavenumbers $\left(1620 \mathrm{~cm}^{-1}\right.$, amide I; and $1514 \mathrm{~cm}^{-1}$, amide II), mainly in the films spectra (Figure 4a). 

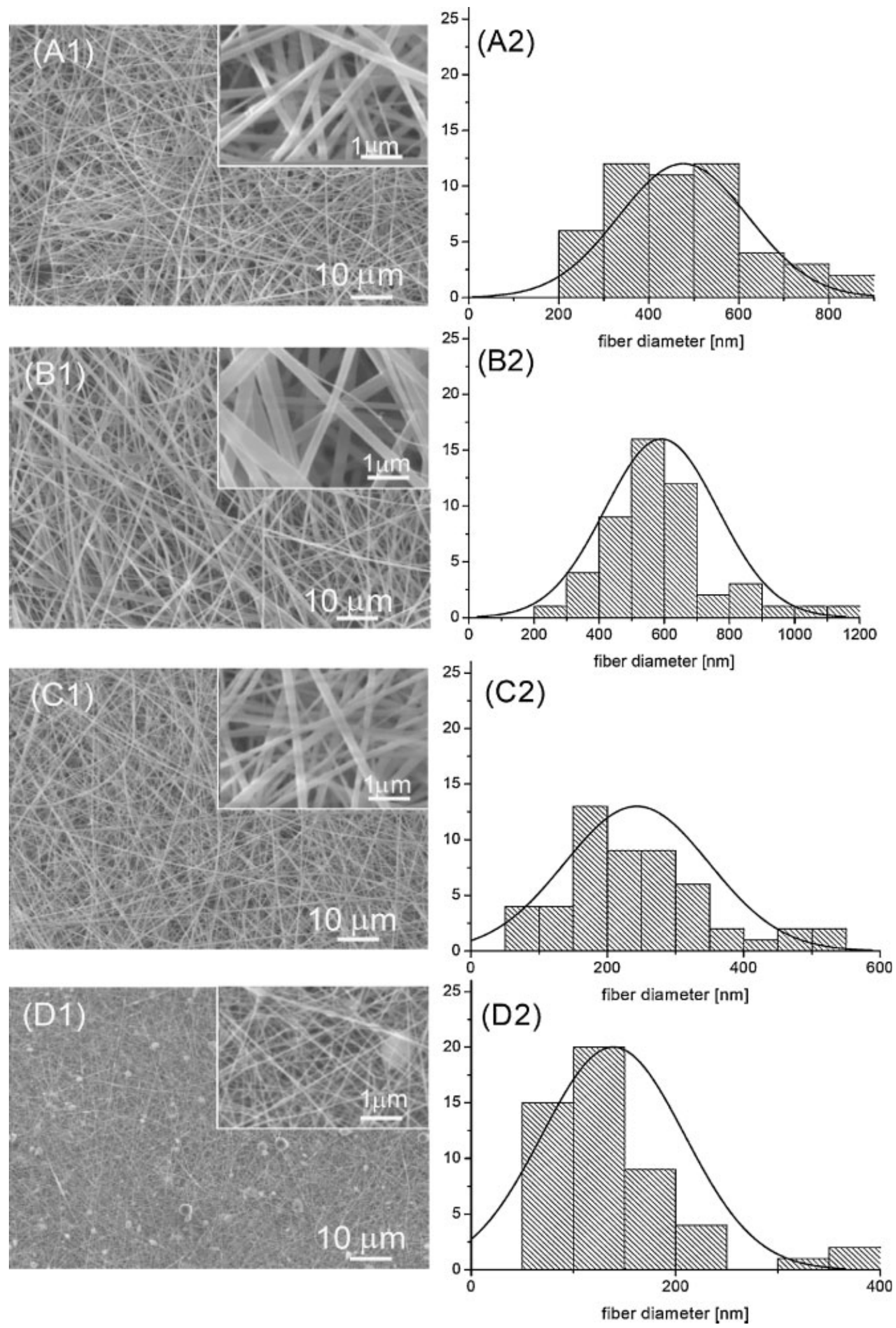

Figure 2. SEM micrographs of as-prepared SF and SFGE nanofibers: $\left(A_{1}\right)$ SF nanofibers, and $\left(A_{2}\right)$ the corresponding histogram of the fiber-diameter distribution; (B1) nanofiber SFGE2, and (B2) the corresponding histogram of the fiber-diameter distribution; ( $\left.C_{1}\right)$ nanofiber SFGE15 and, (C2) the corresponding histogram of the fiber-diameter distribution; (D1) nanofiber SFGE24 and, (D2) the corresponding histogram of the fiber-diameter distribution. The insets are high-magnification SEM micrographs. 

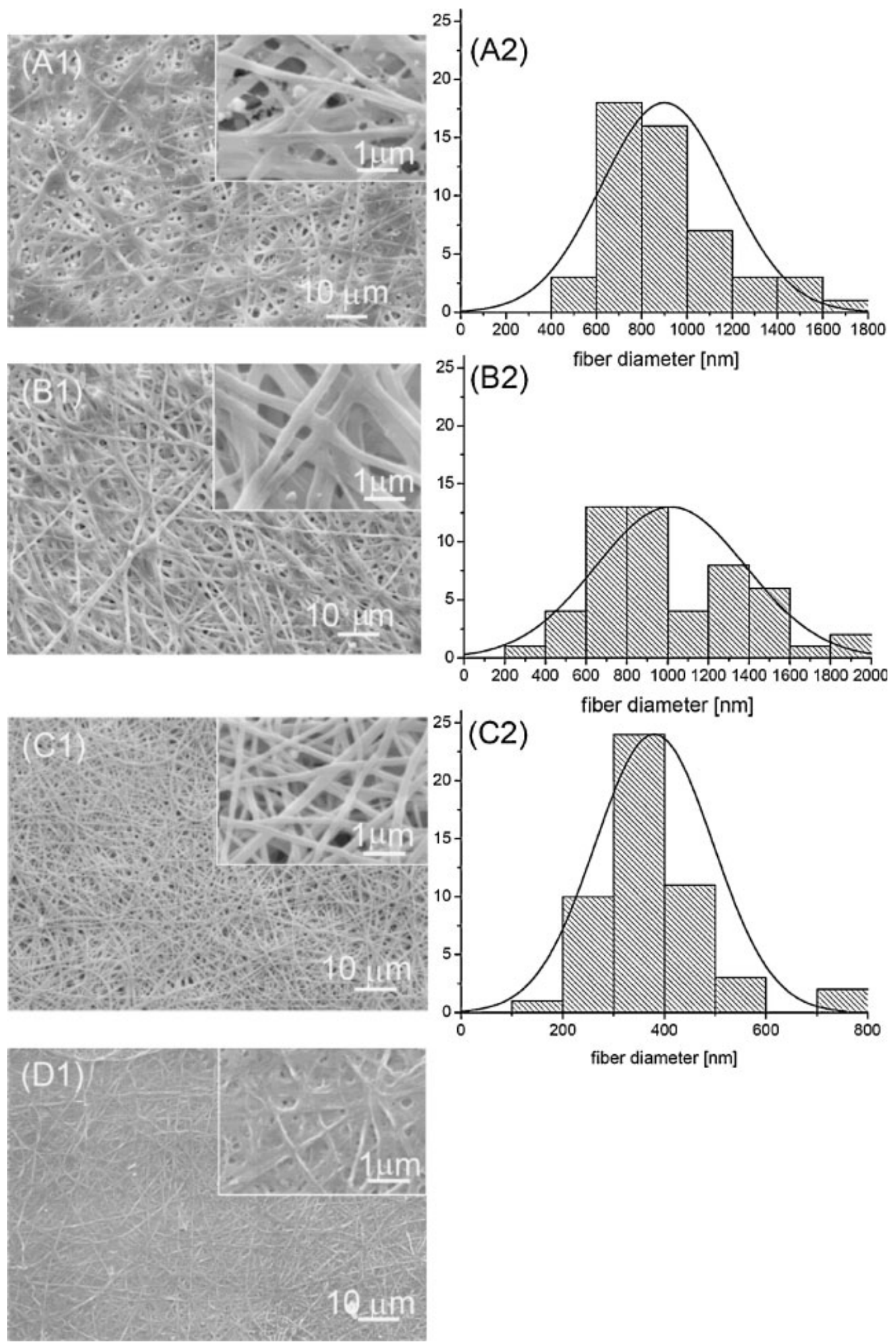

Figure 3. SEM micrographs of SF and SFGE nanofibers after $8 \mathrm{~d}$ immersed in distilled water at $37^{\circ} \mathrm{C}:\left(\mathrm{A}_{1}\right) \mathrm{SF}$ nanofibers and (A2) the corresponding histogram of the fiber-diameter distribution; (B1) nanofiber SFGE2, and (B2) the corresponding histogram of the fiberdiameter distribution; ( $\left.\mathrm{C}_{1}\right)$ nanofiber SFGE15 and, $\left(\mathrm{C}_{2}\right)$ the corresponding histogram of the fiber-diameter distribution; (D1) nanofiber SFGE24. The insets are the high-magnification SEM micrographs. All of the samples were dried in vacuum for 1 week prior the SEM observations. 

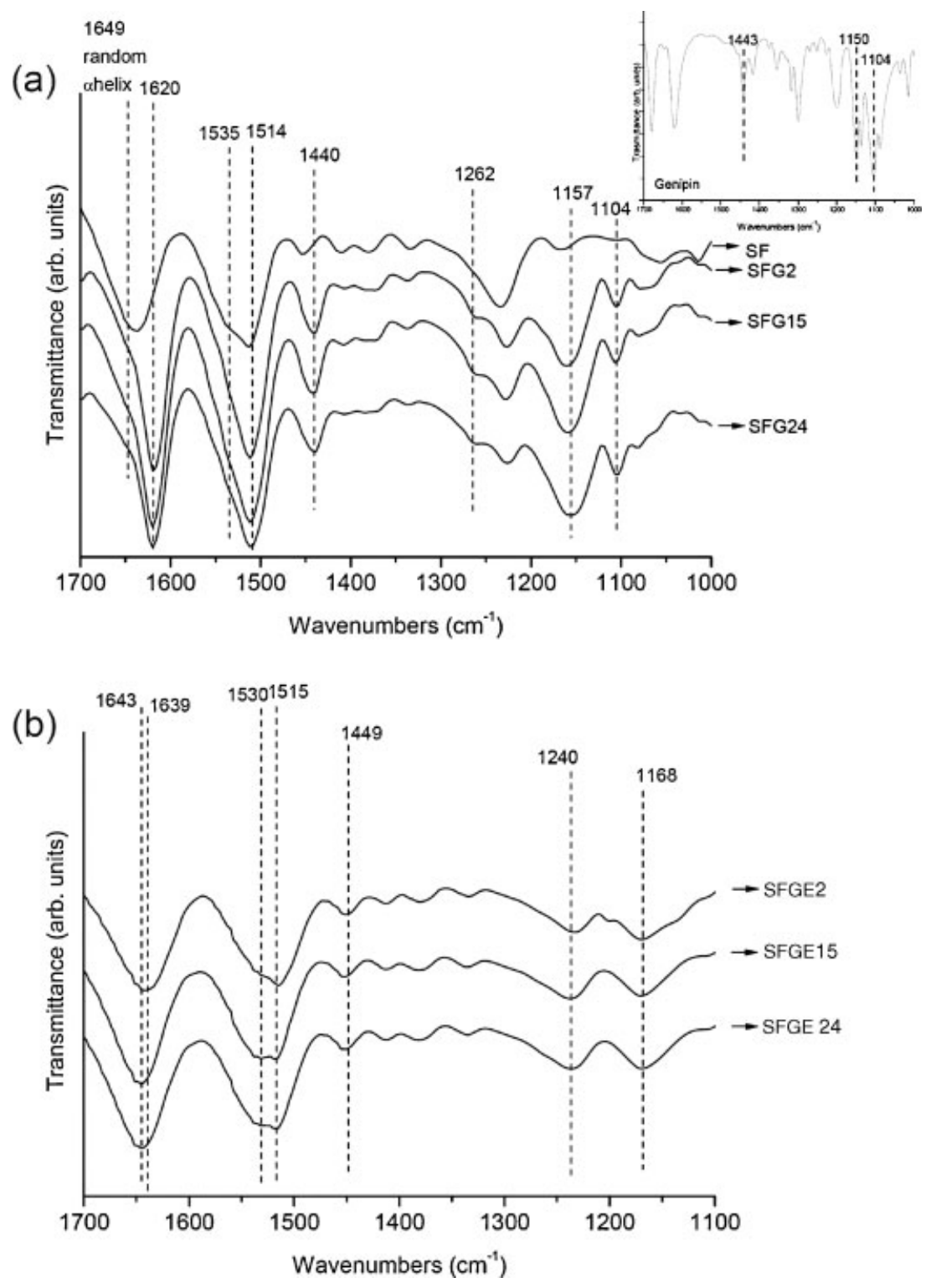

Figure 4. FTIR spectra of the samples: (a) SF and SFGE films, and (b) SFGE nanofibers. The insert in (a) shows the genipin spectrum.

These peaks in the SFGE samples suggest the presence of a $\beta$-sheet structure (silk II structure). Furthermore, some characteristic bands of genipin at $1443\left(-\mathrm{CH}_{3}, \mathrm{CH}_{2}\right), 1150$ $(-\mathrm{COH})$ and $1105(-\mathrm{COH}) \mathrm{cm}^{-1}$ (Figure 4, inset) ${ }^{[24]}$ appeared displaced in the spectra (Figure 4). These displacements could be attributed to modification of SF molecules due to the reaction with genipin. When modified SF solutions undergo electrospinning, further conformational changes may occur, with a further transition of random coils to a more-stable, antiparallel $\beta$-sheet conformation throughout intermediate forms such as $\beta$-turns and $\beta$-bends. To confirm this change, ATR-FTIR spectra of SF nanofibers were examined more carefully in the amide I region
(1700-1650 $\mathrm{cm}^{-1}$ ) - see Figure 5. The amide I spectral region was resolved into three or four main components for each sample, assuming a Gaussian behavior for each individual process. The curve-fitting results (Figure 5 and Table 2) showed that SFGE nanofibers have contributions of different conformations such as random coil (1640$\left.1648 \mathrm{~cm}^{-1}\right), \alpha$-helix $\left(1651-1657 \mathrm{~cm}^{-1}\right), \beta$-sheet $(1625-$ $\left.1640 \mathrm{~cm}^{-1}\right)$, and some intermediate forms such as turn and bends $\left(1658-1666 \mathrm{~cm}^{-1}\right) \cdot{ }^{[22,23]}$ The addition of genipin and/or the electrospinning process promoted particular conformational changes in the silk-fibroin secondary structure of each sample. In particular, in the SFGE15 sample, the frequency of the peaks (92.4\%) 

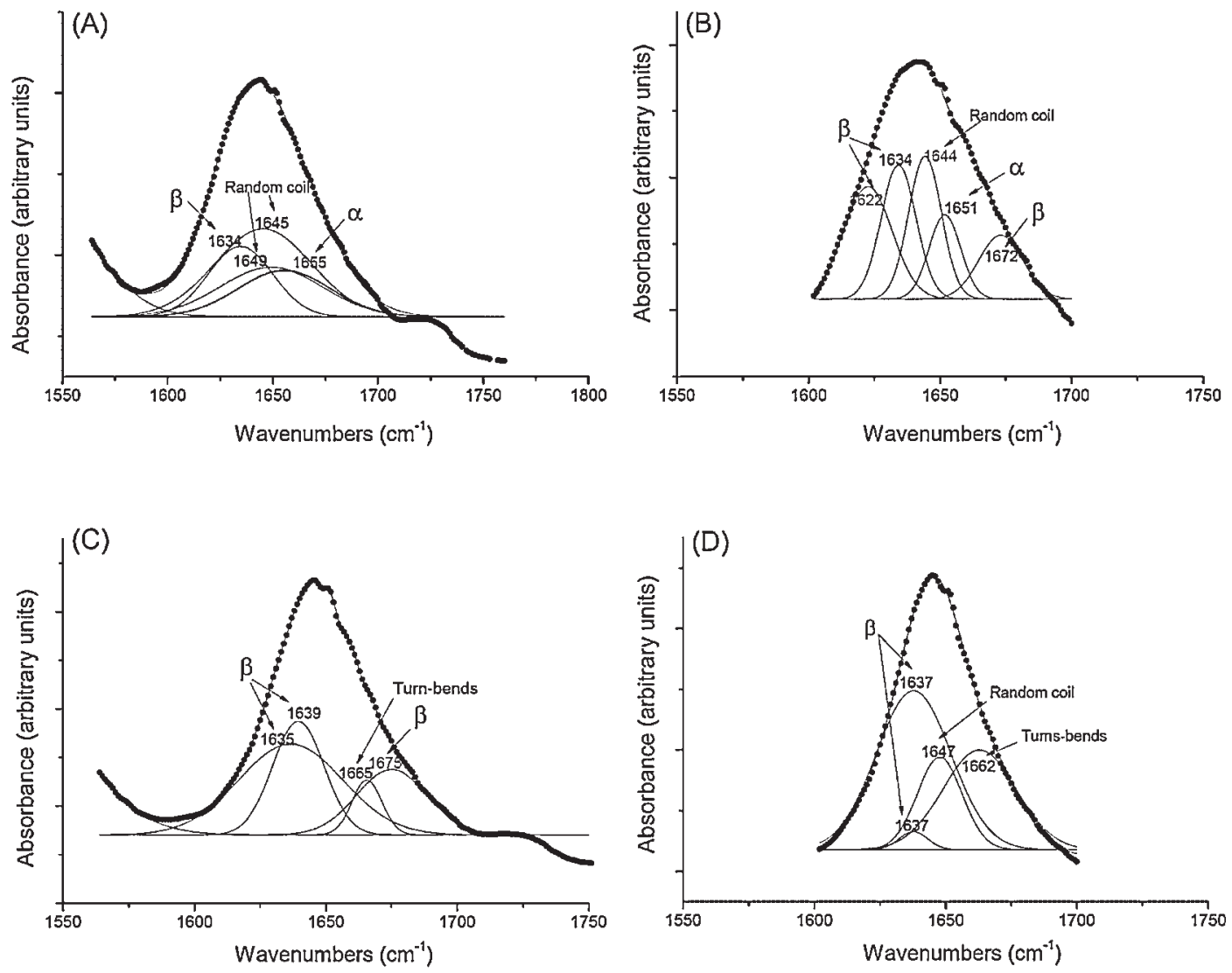

Figure 5. Curve-fitting results of the ATR-FTIR spectroscopy data in the amide II region for the silk-fibroin nanofibers: (A) SF, (B) SFGE2, (C) SFGE15 and (D) SFGE24.

Table 2. Curve-fitting results of "amide I" regions in the ATR-FTIR spectra of the SF nanofibers.

\begin{tabular}{|c|c|c|c|}
\hline \multirow[t]{2}{*}{ Sample } & \multirow{2}{*}{$\frac{\text { Wavenumber }}{\mathrm{cm}^{-1}}$} & \multirow{2}{*}{$\frac{\text { Peak area (Percentage total pick area) }}{\%}$} & \multirow[t]{2}{*}{ Secondary structure ${ }^{a)}$} \\
\hline & & & \\
\hline SF & 1634 & 19.7 & $\beta$-structure \\
\hline$"$ & 1645,1649 & $38.5,23.1$ & Random coil \\
\hline$"$ & 1655 & 18.7 & $\alpha$-helix \\
\hline SFGE2 & 1622 & 26.7 & $\beta$-structure \\
\hline$"$ & 1634 & 23.3 & $"$ \\
\hline$"$ & 1672 & 13.9 & $"$ \\
\hline$"$ & 1644 & 23.3 & Random coil \\
\hline$"$ & 1651 & 12.8 & $\alpha$-helix \\
\hline SFGE15 & 1635 & 44.3 & $\beta$-structure \\
\hline$"$ & 1639 & 26.6 & $"$ \\
\hline ", & 1675 & 21.5 & $"$ \\
\hline ", & 1665 & 7.6 & Turns and bends \\
\hline SFGE24 & 1637 & 51.9 & $\beta$-structure \\
\hline$"$ & 1647 & 17.3 & Random coil \\
\hline ", & 1662 & 30.8 & Turns and bends \\
\hline
\end{tabular}

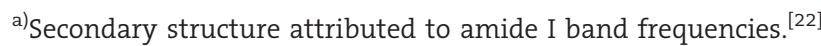


ascribed to the $\beta$-sheet structure, as well as turns and bends structures (7.6\%), revealed a more-stable structure compared to others samples (Table 2). Additionally, the presence of a small percentage of random coils was observed, mainly in the SFGE2 (23.3\%) and SFGE24 (17.3\%) samples. The higher percentage of $\beta$-sheets found in the modified SF nanofibers supports the hypothesis that SF molecules were structurally rearranged by addition of genipin, as well as by the processing effect. The findings also suggested that the SFGE15 system represents the optimal condition for the formation of SFGE nanofibers, in terms of structure and water stability.

\section{Conclusion}

Nanometric nets were successfully obtained by electrospinning regenerated silk fibroin-genipin (SFGE) solutions. The resulting SFGE nanofibers had a fiber diameter from 140 to $590 \mathrm{~nm}$ and were generally thinner than nonmodified SF nanofibers. SFGE15 nanofibers maintained their integrity in water even after $8 \mathrm{~d}$ of aging. The evaluation of the secondary structure of SFGE nanofibers revealed the presence of both $\beta$-turn intermediates and a $\beta$-sheet conformation, induced by the reaction of silk fibroin with genipin. This result was more evident in the fibers produced from the SFGE15 and SFGE24 solutions. The water stability and structure of the investigated SFGE nanofibers makes the process suitable for the fabrication of fibroin nets that do not need any stabilization treatment after spinning, and that could be used in a variety of biomedical applications, such as wound dressings or, with appropriate modifications, for tissue engineering.

Acknowledgements: We gratefully acknowledge Prof. Rolando Barbucci (Department of Chemical and Biosystem Sciences and Technologies, University of Siena, Italy) for assistance with the FTIR-spectroscopy studies. The authors acknowledge funding from the Portuguese Foundation for Science and Technology (FCT), Portugal for SFRH/BD/8658/2002 and the EU-funded Project HIPPOCRATES (NMP3-CT-2003-505758). This work was carried out under the scope of the European NOE EXPERTISSUES (NMP3-CT-2004-500283).

Received: November 16, 2007; Revised: February 28, 2008; Accepted: March 3, 2008; DOI: 10.1002/mabi.200700300
Keywords: biofibers; electrospinning; genipin; modification; silk fibroin

[1] M. Y. Li, M. J. Mondrinos, M. R. Gandhi, F. K. Ko, A. S. Weiss, P. I. Lelkes, Biomaterials 2005, 26, 5999.

[2] O. P. Pham, U. Sharma, A. G. Mikos, Tissue Eng. 2006, 12, 1197.

[3] S. Ramakrishna, K. Fujihara, W. E. Teo, T. C. Lim, Z. Ma, "An Introduction to Electrospinning and Nanofibers", World Scientific, Singapore 2005, p. 1.

[4] R. Murugan, S. Ramakrishna, Tissue Eng. 2006, 12, 435.

[5] N. Bhattarai, D. Edmondson, O. Veiseh, F. A. Matsen, M. Zhang, Biomaterials 2005, 26, 6176.

[6] J. W. Lu, Y. L. Zhu, Z. X. Guo, P. Hu, J. Yu, Polymer 2006, 47, 8026.

[7] Z. M. Huang, Y. Z. Zhang, S. Ramakrishna, C. T. Lim, Polymer 2004, 45, 5361.

[8] L. Buttafoco, N. G. Kolkman, P. Engbers-Buijtenhuijs, A. A. Poot, P. J. Dijkstra, I. Vermes, J. Feijen, Biomaterials 2006, 27, 724

[9] H. J. Jin, S. V. Fridrikh, G. C. Rutledge, D. L. Kaplan, Biomacromolecules 2002, 3, 1233.

[10] J. Ayutsede, M. Gandhi, S. Sukigara, M. Micklus, H. E. Chen, F. Ko, Polymer 2005, 46, 1625.

[11] B. M. Min, L. Jeong, K. Y. Lee, W. H. Park, Macromol. Biosci. 2006, 6, 285.

[12] C. Vepari, D. L. Kaplan, Prog. Polym. Sci. 2007, 32, 991.

[13] E. Servoli, D. Maniglio, A. Motta, R. Predazzer, C. Migliaresi, Macromol. Biosci. 2005, 5, 1175.

[14] R. E. Unger, K. Peters, M. Wolf, A. Motta, C. Migliaresi, J. Kirkpatrick, Biomaterials 2004, 25, 5137.

[15] M. Santin, A. Motta, G. Freddi, M. Cannas, J. Biomed. Mater Res. 1999, 46, 382.

[16] K. Ohgo, C. H. Zhao, M. Kobayashi, T. Asakura, Polymer 2003 44,841

[17] C. Chen, C. Chuanbao, M. Xilan, T. Yin, Z. Hesun, Polymer 2006, 47, 6322

[18] M. F. Butler, Y. F. Ng, P. D. A. Pudney, J. Polym. Sci., Part A Polym. Chem. 2003, 41, 3941.

[19] H. W. Sung, I. L. Liang, C. N. Chen, R. N. Huang, H. F. Liang, J. Biomed. Mater. Res. 2001, 55, 538.

[20] H. W. Sung, R. N. Huang, L. L. H. Huang, C. C. Tsai, C. T. Chiu, J. Biomed. Mater. Res. 1998, 42, 560.

[21] H. M. Chen, O. Y. Wei, L. Y. Bisi, C. Martoni, S. Prakash J. Biomed. Mater. Res. Part A 2005, 75A, 917.

[22] D. Wilson, R. Valluzi, D. Kaplan, Biophysical J. 2000, 78, 2690.

[23] K. K. Chittur, Biomaterials 1998, 19, 357.

[24] F. L. Mi, S. S. Shyu, C. K. Peng, J. Polym. Sci., Part A: Polym Chem 2005, 43, 1985.

[25] I. C. Um, H. Y. Kweon, K. G. Lee, Y. H. Park, Int. J. Biol. Macromol. 2003, 33, 203. 